\title{
XXVI. Some variations observed in electroscopic measurements and their prevention
}

\author{
A.S. Russell M.A. B.Sc.
}

To cite this article: A.S. Russell M.A. B.Sc. (1910) XXVI. Some variations observed in electroscopic measurements and their prevention , Philosophical Magazine Series 6, 19:110, 278-284, DOI: $10.1080 / 14786440208636799$

To link to this article: http://dx.doi.org/10.1080/14786440208636799

曲 Published online: 21 Apr 2009.

Submit your article to this journal $\sqsubset$

Џ Article views: 3

Q View related articles $\square$ 
within a cone of small angle, cutting off but a small portion of terrestrial surface near the oscillator, true diffraction-bands are found, arising ultimately from terms, now important, in which order and argument are nearly equal. These bands are also in accord with expectation. But within the shadow, beyond the extreme generators of the cone, the extinction of the waves is very complete. The harmonics of high order are found to be so disposed as to neutralize one another in a remarkable way, and the intensity of the diffracted light, at a distance of a few thousand miles round the surface, sinks to a minute fraction of its value when the sphere is absent. Thus it is improbable that diffraction can explain the effects, unassisted by reflexion from an ionized layer in the upper atmosphere, or by some other cause. The use of directive oscillators in practice, can cause a change, and Sommerfeld* has shown that the finite conductivity of the earth may be sufficient.

There is one other point of importance in which M. Poincaré's investigation may be criticised. The harmonic series is, at one point, replaced by a definite integral in the ordinary way, in order to obtain its first approximation. But there is a special class of series, to which this belongs, for which this integral is not valid. This is the class whose general term contains an exponential of very large argument. The proper formula, expressed as in the integral, for this case is very different, and has been given by the writer $\dagger$ to a high degree of approximation, having also been originally suggested to him by problems of diffraction by large obstacles.

The proof of the physical results mentioned in the course of this note will be given in a later communication.

XXVI. Some Variations observed in Electroscopic Measurements and their Prevention. By A. S. Russelu, M.A., B.Sc., Carnegie Research Scholar and 1851 Exhitition Scholart.

TWHE curious varying-leak effect observed in working with 1 the $\gamma$ rays of radium and uranium $X$ already described (Soddy and Russell, Phil. Mag. Oct. 1909, p. 635, last paragraph), has now been investigated in some detail and has been traced primarily to the use of too long a stick of sulphur in insulating the leaf system.

As the work shows clearly a possible source of error to be

* Ann. der Phys. 16th March, 1009.

+ Messenger of Mathematics, October 1907.

I Communicated by F. Soddy, M.A. 
avoided in the construction of gold-leaf electroscopes, and as some of the effects obtained are interesting in themselves, a short account of them may prove useful.

In a cylindrical electroscope of the ordinary type containing a gold-leaf system insulated by a sulphur rod, if the time for the leaf to cross the scale in the eyepiece of the microscope was taken and the experiment repeated a number of times, the rate of leak gradually increased until a maximum rate of leak was established. This effect was obtained with both radium and uranium $X$ as the ionizing source and with both $\beta$ and $\gamma$ rays. The effect was obtained also, when the leaf was charged continuously either negatively or positively and in $\mathrm{CO}_{2}$ as well as in air. The effect was shown to depend on the presence or absence of a charge on the leaf system ; for if the charge and the ionization were maintained, the rate of leak tended towards a maximum; while if the ionization was maintained for a sufficient period after the charge had been dissipated, the rate of leak became a minimum.

In the initial observations of this varying-leak effect, the gold leaf used throughout had been charged to a potential rather higher than advisable. The fact that the cause of the effect might possibly be due to a high potential of the leaf, suggested the weighting of an ordinary gold leaf by a short piece of $.025 \mathrm{~mm}$. platinum wire. By doing this a much higher potential is obtained for a given divergence of the leaf than with an ordinary gold leaf. The weighted leaf was found to exaggerate the effect previously obtained and to bring to light others which, by their smallness, had not been brought out' before.

Fig. 1 (p.280) is a curve which shows graphically some of the effects obtained. The rate of discharge of the weighted leaf in scale-divisions per minute as ordinate, is plotted against the time at the commencement of each leak from the start of the experiment, as abscissa.

The electroscope had not been used for some time and the leaf was discharged. The leaf was charged up negatively by means of a charging rod, and the rate of discharge of the leaf under the $\gamma$ rays of the constant ionizing source measured. A represents the leak in scale-divisions per minute corrected for natural leak. The leaf was immediately recharged to the same negative potential and the rate of discharge measured again. Continuation of this process gave the values $A_{2}, A_{3}, A_{4}$, etc., each being larger than the one before, until successive chargings gave no further increase. B represents the leak at this point which 
280 Mr. A. S. Russell on some Variations olserved in

now continues constant if the successive measurements are properly performed. B was usually from 20 to 30 per cent. greater than A. At this point, instead of being charged negatively further, the leaf was charged positively, the

Fig. 1.

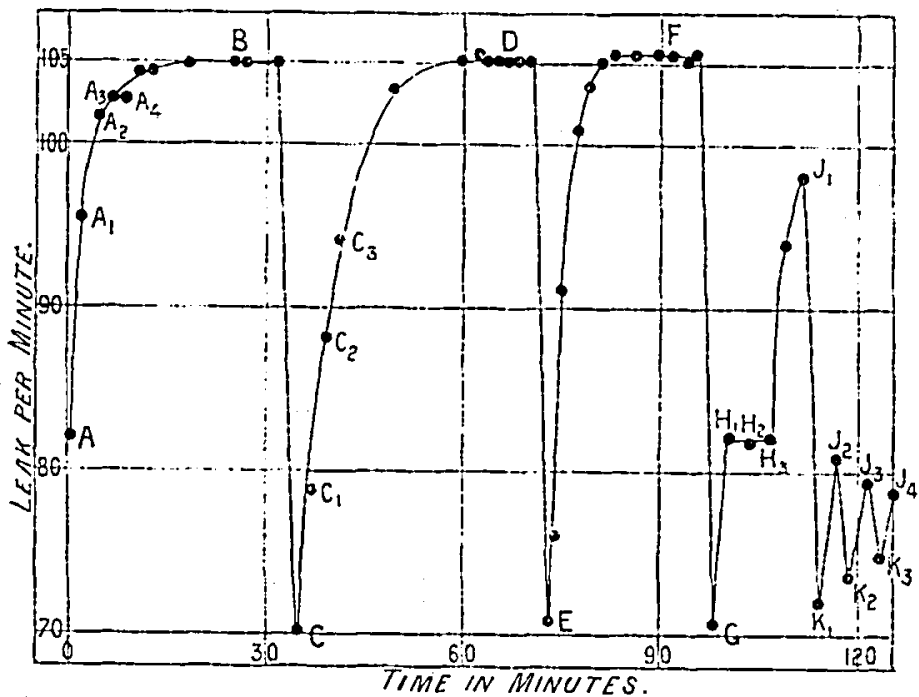

absolute value of the potential being the same. The leak $\mathrm{C}$ obtained is now less than $A$ by about the same amount as B is greater than A. Repeated charging positively gives increasing leaks $\mathrm{C}_{1}, \mathrm{C}_{2}, \mathrm{C}_{3}$, passing through the original value $A$ and finally attaining a steady maximum $D$. The value of the leak at $D$ is, within the limit of experimental error, the same as at B. After charging positively at $D$ and getting a leak which neither increased nor decreased, the leaf was charged negatively. The leak immediately dropped to $\mathbf{E}$ and is equal to the value of the leak at C. Further charging negatively gave a continuous increase in the leak till a point $F$ is reached at which, as at $B$ and $D$, the leak is steady. At $F$, as before, the sign of the charge was reversed and the leak immediately fell off to a value represented by $G$. At this point the air in the electroscope was thoroughly ionized for 30 seconds by $6.7 \mathrm{mg}$. of radium bromide and the leak immediately taken in the usual way. A value $H_{1}$ was obtained equal to the original value $A$ and intermediate between the maximum and minimum values. This was exactly repeated $i$ wice, the first time, $\mathrm{H}_{2}$, the leaf being charged negatively, and the second, $\mathrm{H}_{3}$, as in $\mathrm{H}_{1}$, positively. 
The values $\mathrm{H}_{2}$ and $\mathrm{H}_{3}$ obtained are both equal to $\mathrm{H}_{1}$ and $A$. The leak obtained thus is referred to as the normal leak. Leaks such as B, D, or F obtained after repeated charging of the leaf to the same potential of one sign are denoted as maximum leaks. Leaks $\mathrm{C}, \mathrm{E}$, and $\mathbf{G}$ obtained by altering the sign of the potential at the maximum points are denoted as minimum. The maximum leaks, whether obtained by positive charging or negative are all equal and so are the minimum leaks.

The effect of altering the sign of the charge at any point was next tried. At a point such as $J_{1}$, which is between the normal and the maximum leak, if the sign of the potential be reversed, the leak falls to $K_{1}$, which, however, is greater than the minimum leak. It was found that the nearer the point $J$ is to the maximum the nearer will $K$ be to the minimum and vice versa. The same holds also for a point between the normal and the minimum leaks.

The curve $J_{1}, K_{1}, J_{2}, K_{2}, J_{3}, K_{3}, J_{4}$ shows this. The graph of a succession of readings taken by charging alternately positively and negatively therefore would be an oscillatory curve in which the oscillations get smaller and smaller until they cease altogether. This would hold true if the charging up each time were done perfectly, but slight variations in

Fig. 色.

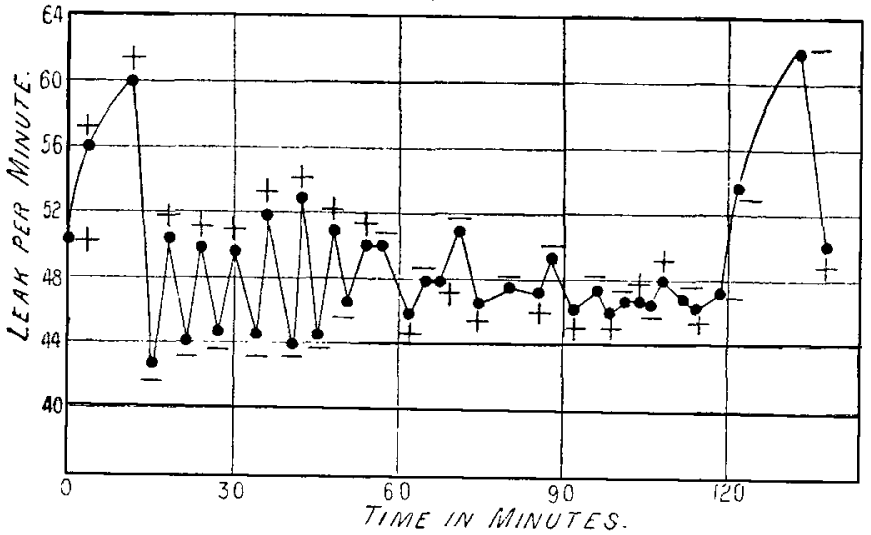

this, which will be discussed later, cause variations which prevent the oscillatory curve from developing into a straight line. Some results illustrative of this are plotted in fig. 2, where the initial and final leaks are normal leaks.

Ionization by an external source preceding charging, provided it is intense enough, always gives the normal leak. 
The normal leak is obtained also by weak ionization over a correspondingly longer time, and indeed the natural ionization of the electroscope's air is itself sufficient if the whole instrument be left for 4 to 6 hours. It is not necessary that the leaf be charged. The normal leak because of its constancy for any one disposition, and for the ease and rapidity with which it may be obtained, was used throughout in the work on the absorption of the $\gamma$ rays of uranium $X$ and radium. The effects described have been regularly and clearly shown though in varying degree by every system whether weighted or not, tried in electroscopes of the ordinary pattern. It is immaterial (1) what is the nature of the electroscope walls or base ; $(2)$ what is the nature of the ionizing source, whether it be $\beta$ or $\gamma$ rays, or from radium or uranium ; (3) whether the source be intense, or made weak by absorbing screens; (4) whether the potential be just great enough to ensure saturation or much greater. If $\Delta_{1}$ denotes the percentage of maximum leak over normal leak, and $\Delta_{2}$ that of normal over minimum, a series of experiments has proved that:

(1) $\Delta_{1}$ is much greater for a weighted leaf than for an ordinary gold leaf, varying from 4 per cent. with the first to 38 per cent. in the second for typical cases;

(2) for an unweighted gold leaf, $\Delta_{1}$ increases as strength of source decreases, for a given potential ; also $\Delta_{1}$ is practically constant between a divergence of tip of leaf from its discharged position from $7 \mathrm{~mm}$. to $13 \mathrm{~mm}$.

For a weighted leaf :

(1) $\Delta_{1}$ varies in same direction as potential of leaf if strength of source be constant: thus,

Source $=50$ div, per $\min$.

\begin{tabular}{|c|c|c|c|c|c|c|}
\hline Potential in rolts & 300 & 350 & 400 & 460 & 530 & 600 \\
\hline$\Delta_{1} .$. & $21 \cdot 3$ & 205 & $27 \cdot 8$ & $28 \cdot 0$ & $30 \cdot 2$ & $35 \cdot 8$ \\
\hline
\end{tabular}

(2) $\Delta_{1}$ decreases as strength of source, $S$, increases, if potential be constant :

Potential 460 volts.

\begin{tabular}{|c|c|c|c|c|c|c|}
\hline $\mathbf{s}$ & $8 \cdot 40$ & $12 \cdot 98$ & $17 \cdot 36$ & 46.9 & $48 \cdot 3$ & 800 \\
\hline & $38 \cdot 4$ & 322 & $30 \cdot 4$ & 28.0 & 27.9 & $28 \cdot 0$ \\
\hline
\end{tabular}


Similar results were obtained for potentials of 400 and 350 volts.

(3) $\Delta_{2}$ always varies directly as $\Delta_{1}$, decreasing as strength increases for constant potential, and increasing with potential for a constant strength of source.

Subsidiary effects as well as the chief effect are shown particularly with unweighted leaves where they are not masked so much by the chief effect. The magnitude of any leak for one fixed disposition varies slightly (1 to 3 per cent.) according as (1) the leaf is charged so that it comes on to the scale in the eyepiece within a short time, say 15 to 30 seconds; the leaf is charged up too much, and (2) it is brought almost on to the scale by an extra source of radium which is then removed; or (3) it is allowed to come on to the scale under the influence of the ionization which is being measured, taking about 2 to 4 minutes to do so. Method (1) was used generally and in making all measurements shown in fig. 1 . The effect of (2) is, as would be expected, to give a leak slightly less than that obtained by (1). Thus a normal leak obtained by charging up as in (2) would be slightly slower than that made in the ordinary way. The effect of $(3)$ is the reverse of (2). In general the leaks obtained by successively charging up to the same potential according to (1) lay on a smooth curve extending from the normal to the maximum

Fig: 3.

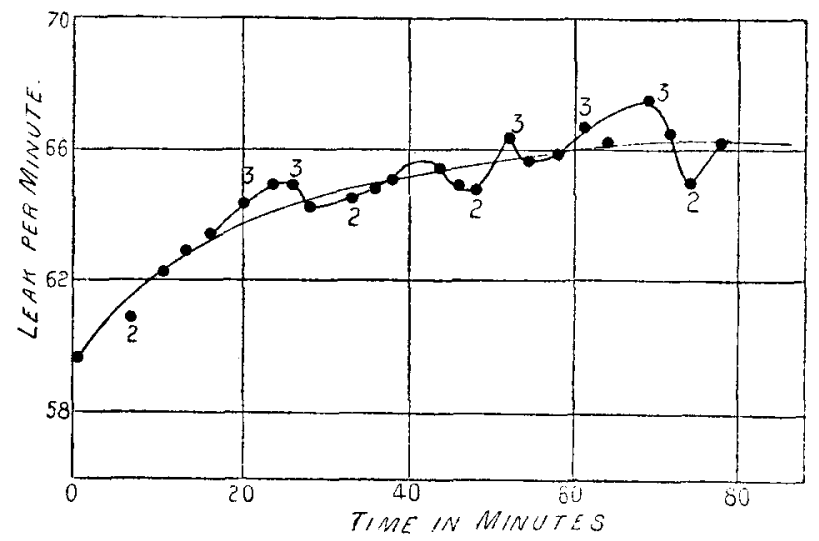

leak, such as shown in fig. 3. The influence of the methods (2) and (3) was to give a point below the smooth curve and above it respectively. After making such a measurement 
284 Variations observed in Electroscopic Measurements.

the next made in accordance with (1) lay on the opposite side of the curve, while further measurements made by (1) caused points to resume their place on the smooth curve. In fig. 3, the points obtained by (2) and (3) are marked. The points not marked were obtained by (1).

The reason for the existence of these effects was sought next. It was found that the placing of an earthed ring round the sulphur, as employed by C. T. R. Wilson in his original work on the subject of electroscopes (Proc. Roy. Soc. 1901, lxviii. p. 152), minimized the effect. Leaf systems, however, were made up with weighted leaves and no earthed ring, which gave the effect considerably less than other leaf systems. It was found eventually that only the leaf systems containing a sulphur rod from 2.5 to $4.4 \mathrm{~cm}$. in length gave the effects, leaf systems with a small bead of sulphur only, with or without the earthed ring, hardly showing the effect at all. A wejghted leaf system with a rod of sulphur $3.5 \mathrm{~cm}$. long as an insulator was made up, the sulphur being completely surrounded by an earthed cylinder of brass. The varying-leak effects were not shown. On raising the ring slightly so that part of the sulphur was left bare the effects were still not shown. On the ring being raised, however, so much that the whole of the sulphur rod was exposed, the effects made themselves felt at once. The effects are thus due to the presence of a long rod of sulphur, the longer the rod, the more pronounced being the effects, and is favoured by a high potential on the leaf. They can be minimized by using a small bead only, and with this an earthed tube is hardly necessary. The effect can be eliminated by thorough ionization of the gas in the electroscope between the measurements. In taking decay curves, or absorption curves, when the strength of the source varies over a wide range, the effects described are especially to be avoided. Apart from them the instrument appears to measure reliably and proportionately the whole range of rates of leaks it is possible to time accurately with a fairly high magnification.

I take this opportunity of thanking Mr. Soddy for his kindness throughout the course of the work and for many valuable suggestions.

Plysical Chemistry Laboratory,

University of Glasgow, December 1909. 